\title{
To The Teacher
}

Many teachers who have had a course in the teaching of a second language are familiar with Robert Lado's Language Teaching, A Scientific Approach and Nelson Brooks' Language and Language Learning. Both contain a wealth of excellent suggestions for the teaching of a second language.

In some situations a native speaker who has not taken a course in the teaching of a second language may be asked to teach a class in his native tongue because no one else is available. A person caught in such a predicament will find the books mentioned above very helpful. Because these books offer so much help, we shall confine our remarks to a few pertinent facts on general procedure and comments on the material in this volume and its use.

Of the points stressed in the brief section addressed to the student, two will be considered here. The first is the importance of memorizing the material. The second is the need to render the student's first language inoperative. Memorization is important because in order to converse in any language one needs automatic recall of the expression needed at any given moment. Fluency in the use of the second language is developed more readily if the first language is submerged during the learning period.

Many experienced teachers, trained in second language teaching, ban the use of the first language during the class sessions. Others limit the use of the first language to giving instructions at the beginning and making comments at the close of a class session. The rest of the class period is devoted entirely to work on the target language without using the first language. Students are not allowed to ask questions. They cannot, if the teacher outlaws the use of the first language!

For many students certain sounds in a new language will cause considerable difficulty. In some cases there may be difficulty with final vowels. This is true especially in Hawaiian, a language in which every word ends with a vowel which must be sounded. It is often difficult to differentiate properly between some final vowels-for example, final $i$ and final $e$; final $o$ and final $u$. Some people do not hear any difference at all. Minimal pairs practice can help to 
solve this problem. The minimal pairs should be practiced when pertinent pronunciation problems arise in learning the utterances.

Word linkage is often a problem also. In this volume a list is provided after each set of basic utterances. Each set of words to be linked should be handled in context and should be modeled carefully. For example, if the utterance is

\section{E hele ana au.}

a. Model the entire utterance several times, being careful to link the words properly but without exaggeration.

b. Model hele ana and have the class imitate. Do this several times.

c. Model hele ana $a u$ and repeat the procedure suggested in b.

d. Model the entire utterance several times and have the class repeat.

When a long utterance is to be taught, it is better to divide it into sections and to teach one section at a time. The procedure followed in some series is to begin with the last phrase or last few words in an utterance which make sense as a group, and then add on what precedes until an entire utterance has been learned. Notice that if taught in this manner, the class will always stop at the end of an utterance. Sometimes, however, this procedure may not prove feasible. Therefore, long utterances should be studied beforehand and possible pauses or divisions be determined in advance. This is important to class success and morale.

Sometimes inexperienced teachers feel that individual recitation takes too much time and causes students to lose interest. Advance planning can be the key to success here.

The seating arrangement can be of great help in expediting individual recitation. Students may be arranged in a square, circle, or semicircle. Any of these arrangements, together with good preplanning, will facilitate the flow and movement of individual recitation. The teacher can begin and end at any given point in the seating arrangement and vary the routine from time to time.

Sometimes the teacher can begin with himself and move to his right or left and go around the group. This procedure can be used to advantage in roundrobin style practice, in questions and answers, or in directed responses. The teacher can exhibit creativity in planning ways to make the practice interesting and to keep the students alert. Much depends on the teacher.

In some textbooks the study of a language is begun by memorizing short utterances in very brief conversations in each unit. After each set of conversations, there is a pattern practice and material for further practice using the structural patterns found in the brief conversations. In other textbooks the units begin with a set of basic utterances to be memorized, followed by pattern practice material, conversations, and several types of activities for further practice. All are designed to provide practice which will promote a firm grasp 
of the basic utterances and the structural patterns used. Such is the plan for this volume.

The basic utterances are arranged in groups. The grouping of utterances is determined by their content. The teacher may teach all the basic utterances first then proceed to the other parts of a unit, or he may use the following procedure or one similar to it.

Model an utterance. Have the class and individuals imitate. When a group of utterances has been learned, take a quick run through. Model utterance 1, class repeat. Model utterance 2, class repeat. Model utterance 3, class repeat. Model all three, class repeat. Class recite without assistance. Individuals recite without assistance.

For variety, pattern practice material may be taken up and the conversations based on these utterances. This requires careful advance planning and can create a more relaxed and varied situation.

Even though the students have learned the basic utterances and have practiced the pattern practice material, when the conversations are read for the first time, the teacher should model the utterances and the class should imitate. The only difference now is that perhaps one modeling should be sufficient. This is to insure good phrasing and expressiveness.

The practice activities which follow the conversations fall under the heading No ka ho'oma'ama' a hou 'ana aku, and they consist of the following: directed responses; questions and answers; short narratives with questions to be answered in the target language; material for special practice on tenses, sentence expansion, comparative forms, and other features all based on the basic utterances; pattern practice material; and conversations. These are designed to help the student become familiar with the material so that a given stimulus will trigger the proper response. If too much time elapses between stimulus and response, the student has not learned to respond automatically.

In many units, material in narrative form is provided without the benefit of translation. The narrative material, based on familiar structural patterns and vocabulary, provides for further practice. In sections with narrative material, questions which follow help to ascertain the student's grasp of structural material. Students may be encouraged to prepare material in narrative form using familiar structural patterns and vocabulary. Some suggestions for this type of activity are given, but the teacher can assign others.

The days of the week and months of the year are taught indirectly, and no special unit is devoted to them. Learning the days and months can begin during the first lesson with the teacher giving the date. For some time the teacher should give the date, then the students can be asked to do this. 
Some direct reference to and information about Hawaiian culture is presented in structural patterns which are or should be familiar to the student, because of the material covered. When the vocabulary is unfamiliar, the English explanation is given immediately, thus enabling the student to concentrate on the information. The narrative material will provide further practice in comprehension and will pave the way for future work.

While some attention is given to what might be referred to as culture, it must be understood that in second language study learning the language takes precedence over information about the culture. The goal should be to use the language in speaking, reading, and writing. When students gain proficiency in the language, more cultural information is obtainable by reading materials in the second language. Their language proficiency will heighten their understanding of the meanings and connotations of the target language in a way not possible through translations. According to Professor Lado, "A language $\ldots$ is both a component of culture and a cultural network through which the other components are expressed" (1964:23).

In section VII, No ka pilina 'ölelo a me ka papa 'ölelo, some information about the structure of basic utterances is given, but this is not intended for class discussion. All classwork should be conducted for the most part in the target language. Students who are unduly concerned about the word-forword equivalents, and there are some in every class, should read the material in section VII. They will notice that we reinforce what is given in the basic utterances. No literal translation is given because each language has its own structural patterns, and literal translations merely serve to impede progress in learning the target language. 